\title{
Possible Manifestation of Heavy Stable Colored Particles in Cosmology and Cosmic Rays
}

\author{
R. N. Mohapatra ${ }^{1}$ and S. Nussinov ${ }^{2}$ \\ (1) Department of Physics, University of Maryland, College Park, MD-20742, USA. \\ (2) School of Physics and Astronomy, Tel Aviv University, Tel Aviv, Israel \\ and Department of Physics, University of South Carolina, Columbia, SC-29208
}

(August, 1997)

\begin{abstract}
We discuss the cosmological implications as well as possible observability of massive stable colored particles which often appear in the discussion of physics beyond the standard model. We show that if their masses are bigger than few hundred $\mathrm{GeV}$ and they saturate the halo densityand/or they occur with closure density of the universe, they can be ruled out by existing limits on heavy stable particles from analysis of anomalously heavy isotopes of ordinary nuclei as well as from the observations in WIMP detectors. We also derive constraints on their masses if they annihilate to produce gamma rays. We then comment on the possibility that these particles could be responsible for the ultra high energy (UHE) cosmic rays with energies $\geq 10^{20} \mathrm{eV}$ observed in several recent experiments and in particular point out that their low inelasticity argues against the possibility they can explain the observed UHE events.
\end{abstract}

UMD-PP-98-17

\section{INTRODUCTION}

Heavy stable particles carrying gauge charges naturally arise in many field theoretic contexts. Such particles with electric charges have been extensively discussed in the past [1] in connection with their possible role as dark matter. We will assume in what follows that these particles are electrically neutral but carry color. Examples of such particles (to be denoted henceforth by $X$ ) can arise in supersymmetric models where the gluino ( rather than the photino ) is the lightest SUSY particle in which case we will have stable $\tilde{g} g$ bound states with masses in the low TeV range (or much lower masses in the light gluino scenario [2]). There can be examples of gauge mediated supersymmetry breaking models [3] where one can have color octet messengers that can be stable and heavy (with masses easily in the $10 \mathrm{TeV}$ range). There are also examples of mirror models for particle where the particle spectrum as well as the gauge symmetries are completely doubled [4]. These models have been proposed in connection with understanding the neutrino puzzles or to solve the strong CP problem. If in these models the color $S U(3)_{c}$ groups of the mirror universe mixes with the that of the known universe, then the mirror quarks will share the strong interaction but not the observed electroweak interactions. Finally, the well known magnetic monopoles that arise in grand unified theories, although overall color neutral can carry a color cloud in addition to magnetic charge if the initial GUT group is simple.

In this paper, we comment on the cosmological aspects and possible experimental observability of such particles 7 . We use a plausible ansatz for the annihilation as well as scattering cross-section for these particles and draw the following conclusions: (i) if their masses are above several hundred $\mathrm{GeV}$, and they saturate the halo density, they should have been seen in the existing underground detectors and are excluded by present searches [7,8; ; and secondly (ii) in the same mass range they are also excluded by the recent limits on anomalously heavy isotopes of ordinary nuclei [6]. Furthermore, if either the $X$ particles are their own antiparticles (as in the case of color octets or gluinogluon bound states) or the $X$ and $\bar{X}$ are equally abundant ( saturating the halo density in both the cases), their annihilation in the halo can lead to energetic gamma ray fluxes. Present limits on these fluxes rule out the mass range $M_{X} \leq 300 \mathrm{TeV}$.

We also focus on recent suggestions in the literature to utilize particles 9, 10 in the above general category to explain the puzzling phenomena of ultra high energy (UHE) cosmic ray events [11] observed in several independent experiments in the past decade. One could imagine these colored but electrically neutral particles [10] playing the

\footnotetext{
${ }^{1}$ For an earlier analysis of strongly interacting particles which constitute the halo dark matter see G. Starkman et al. [5]. Our work is complementary to this and addresses several issues not covered in Ref. [5]
} 
role of the original high energy particle as the cause of these UHE cosmic rays. Our discussion leading to the above constraints also makes it clear that if these particles have masses above several hundred $\mathrm{GeV}$, their hadronic collisions have very low inelasticity making them unsuitable as the UHE primaries which need to have significant inelasticities in order to generate the observed shower characteristics.

This paper is organized as follows: in section 2, we present estimates of the annihilation, scattering and pair creation cross-sections of such particle. Using these estimates in sec. 3, we proceed to discuss the residual abundance of such particles in the present universe, which we find, could provide the closure density of the universe. In section 4, we discuss their prospects for nuclear capture and formation of rare isotopes and their observability in various experiments. We then comment briefly on the connection between the UHE primaries and the massive $X$ - particles in section 5. In section 6 , we discuss in some detail the suggestion [12] that the magnetic monopoles are responsible for the UHE cosmic ray events and argue that very low inelasticity of monopole-nuclear collisions tend to preclude this mechanism.

\section{REACTION CROSS-SECTIONS FOR THE $X$ PARTICLES}

Because the $X$ particles are much more massive than the usual hadrons, we must assume that their mass originates not from QCD but from physics at some new scale such as the scale responsible for breaking supersymmetry. Consequently we envision a small core for the $X$ particle, of size $O\left(M_{X}^{-1}\right)$ where the bulk of the mass is residing. This specific novel feature distinguishes the $X$ particles from the ordinary hadrons.

In $X$-nuclear or $X X$ low momentum transfer collisions, only gluonic degrees of freedom residing in a hadronic cloud of size $\Lambda_{Q C D}^{-1} \simeq$ Fermi need to be involved. Hence we expect to have generic hadronic cross-sections

$$
\sigma_{X-N} \approx \Lambda_{Q C D}^{-2} \simeq 10 \mathrm{mb}
$$

The same estimate applies also to $X-X$ elastic cross-section. On the other hand in $X-\bar{X}$ annihilation process, the cores of $X$ and $\bar{X}$ particles should overlap and we therefore expect this cross-section to be much smaller:

$$
v \sigma_{X-\bar{X}}^{a n n} \approx \frac{\alpha_{s}^{2}}{M_{X}^{2}} \approx \alpha_{s}^{2}\left(10^{-8}-10^{-10}\right) G e V^{-2}
$$

Likewise, just as for $t \bar{t}$ pair production, $X-\bar{X}$ production cross-section in hadronic collision is also very small:

$$
\sigma_{\text {hadrons } \rightarrow X \bar{X}} \approx \frac{\alpha_{s}^{2}}{M_{X}^{2}} f\left(\frac{M_{X}}{\sqrt{s}}\right)
$$

with $f\left(\frac{M_{X}}{\sqrt{s}}\right)$ representing some further (structure function) suppression.

The above crude estimates of the cross-sections for processes involving the $X$ particles suffice for our discussion and unless the above intuitive arguments are completely off, the constraints obtained for the $X$ particles will remain qualitatively unchanged.

One may wonder whether the interactions of the soft hadronic clouds could modify the $X-\bar{X}$ annihilation crosssection. The soft hadronic interction extends over typical hadronic scale ( say, a Fermi) and also have typical hadronic strength i.e. effective potential depth of order $U_{0} \leq G e V$. If this interaction can pull together the "cores" of the two colliding $X$ particles, then one would expect the $\sigma_{X-\bar{X}}^{a n n}$ to be of the same order as the $\sigma_{X-X}^{e l}$ i.e. of order $\sigma_{\text {hadron }} \approx(F e r m i)^{2}$ rather than the small value (Eq.2). The hadronic cloud interaction is strong enough to pull the "cores" of the $X$ particles when the $X$ particles are sufficiently slow moving, $\beta_{X} \leq \sqrt{\frac{G e V}{M_{X}}}$, with their center of mass kinetic energy less than a $\mathrm{GeV}$. In this case we would expect a significant enhancement in their annihilation cross-section all the way to the value of say nucleon-antinucleon annihilation at low velocities:

$$
\sigma_{X-\bar{X}}^{a n n} \approx \frac{1}{\beta}(F e r m i)^{2}
$$

This estimate will hold for example for relic $X$ particles in the late cool stages of the universe. However the earlier estimate Eq. 2 will hold in the early hot stage when the center of mass kinetic energy of the collision exceeds $U_{0} \approx \Lambda_{Q C D}$ and we can ignore the cloud effect. We will see in the next section that this result has a profound implication for the number density of the relic $X$ particles and their subsequent behaviour.

As we will see in the next section, the freezeout temperature $T^{*}$ depends only logarithmically on $\sigma_{X-\bar{X}}^{a n n}$. The ratio $T^{*} / M_{X}$ is always larger than 0.02 so long as $\sigma^{a n n} \leq(F e r m i)^{2}$. For the value of $M_{X} \sim 1-10 \mathrm{TeV}$ (as is the case we 
are considering), the temperature at decoupling $T^{*} \approx 20-200 \mathrm{GeV}$, is considerably larger than $\Lambda_{Q C D}$ making the enhancement of the cross-section noted above irrelevant for the relic abundance.

\section{RELIC DENSITY OF $X$ PARTICLES}

In the very universe $T \gg M_{X}$, we expect the density of $X$ particles to be comparable to that of other relativistic particles. However as the universe cools below $M_{X}$, the annihilation of the $X-\bar{X}$ pairs dominates over their production gradually decreasing the ratio $n_{X} / n_{\gamma}$, until the freezeout temperature where rate of annihilation becomes slower than the expansion rate of the universe. The annihilation rate is given by $\frac{d n_{X} / d t}{n_{X}} \equiv \sigma^{a n n} v n_{X}$ so that the freezeout temperature is dictated by the following inequality [13:

$$
\frac{\alpha_{s}^{2}}{\pi M_{X}^{2}} \leq \frac{1.66 \sqrt{g^{*}} T^{2}}{M_{P \ell}}
$$

Using next $n_{X} \approx g_{X}\left(\frac{M_{X} T}{2 \pi}\right)^{3 / 2} e^{-\frac{M_{X}}{T}}$, (where $g_{X}$ is the number of degrees of freedom of the $X$ particle: e.g. for a color octet spin zero field, $g_{X}=8$ etc) we can rewrite (Eq. ) in terms of the dimensionless quantity $\xi \equiv \frac{M_{X}}{T}$ as follows:

$$
\frac{2 g_{X}}{(2 \pi)^{5 / 2}} \alpha_{s}^{2} e^{-\xi}=\sqrt{g^{*}} \xi^{-1 / 2} \frac{M_{X}}{M_{P \ell}}
$$

Taking $M_{X} / M_{P \ell}=10^{-16}-10^{-15}, g^{*} \simeq 10$ and $\alpha_{s} \simeq .12$, we find

$$
\xi \approx 1 / 2 \xi+\ln 10^{14}+\ln \frac{2 g_{X} \alpha_{s}^{2}}{(2 \pi)^{5 / 2}} \approx 30
$$

Finally using $n_{\gamma} \approx g^{*} T^{3}$ we have

$$
\frac{n_{X}}{n_{\gamma}}=\frac{1.66 \pi^{3}}{2.4 \sqrt{g^{*}} \alpha_{s}^{2}} \xi \frac{M_{X}}{M_{P \ell}} \approx 170 \xi \frac{M_{X}}{M_{P \ell}} \approx 5 \times\left(10^{-12}-10^{-13}\right)
$$

Using next $\eta_{B} \equiv \frac{n_{B}}{n_{\gamma}} \approx 6 \times 10^{-10}$, we find

$$
\frac{n_{X}}{n_{B}} \simeq 10^{-2}-10^{-3}
$$

Hence the mass densities are in the ratio

$$
\frac{\rho_{X}}{\rho_{B}}=\frac{n_{X} M_{X}}{n_{B} m_{B}}=1-100 \quad\left(\text { for } M_{X} \simeq 1-10 \mathrm{TeV}\right)
$$

Thus we find the nice feature that taking $M_{X}$ in the range of 1-10 TeV suggested by independent considerations naturally results in closure density of the universe. This is of course not an entirely new result but clearly related to the discussion of the lightest supersymmetric particle (LSP) with a corresponding weak annihilation cross-section being the dark matter of the universe.

We will now show that there are severe difficulties with such relic particles if the $X$ have hadronic strength interactions with ordinary matter. Once the temperature $T \leq \Lambda_{Q C D} \simeq .3 \mathrm{GeV}$, the QCD phase transition temperature, the gluon and quark gas disappear. At this hadronic recombination temperature, the purely strogly interacting particles almost decouple from the baryons (and from the rest of the background radiation). Once $\rho_{X} \geq \rho_{\gamma}$, a standard cold dark matter scenario may be implemented: the $X$ particle would start forming structure and galactic halos in particular. Subsequently the potential wells of these particles will trap the dissipating baryons which form the galactic discs. If we assume that the local halo density $\rho \approx 0.3 \mathrm{GeV} /(\mathrm{cm})^{3}$, is generated by our $X$ particles, we will have

$$
n_{X} \simeq 0.3 \mathrm{GeV} / M_{X} \simeq 10^{-5} \mathrm{~cm}^{-3}
$$

This of course significantly higher than the value of relic density mentioned earlier (using $n_{\gamma} \approx 300 \mathrm{~cm}^{-3}$ one finds that $n_{X}^{\text {relic }} \simeq 10^{-10}-10^{-11}$ ). We will now discuss three possible manifestations of the $X$ - particles: (i) in underground detectors ; (ii) via rare isotope formation and (iii) via annihilation in the halo. This discussion will be done for both cases of galactically clustered as well as cosmological, unclustered, $X$ particles. 


\section{MANIFESTATION OF THE $X$ PARTICLES}

\section{A. Wimp detectors and $X$ particles}

Let us first consider issue (i) - namely the possible manifestation of the $X$ particles in underground detectors if the $X$ 's saturate the halo density. Even though the $X$ particles are strongly interacting (unlike the usual wimp candidates for dark matter) their penetration depth in earth or water can vastly exceed that of the normal hadrons. This is so despite the fact that as indicated in sec. II, the typical $X$-nucleus scattering may well be of the size of normal hadronic cross-section- i.e. $\sigma \approx \pi R^{2}(A, Z)$ with $R \simeq 1.2 A^{1 / 3}$ Fermi, the nuclear radius. The reason for this is the large mass mismatch between the $X$ particle and a normal hadron. This implies that we require many collisions

$$
n_{\text {coll }} \approx M_{X} /<m_{(A, Z)}>\simeq \frac{M_{X}}{20 G e V}
$$

(where we have chosen $\left\langle m_{(A, Z)}\right\rangle \approx 20 \mathrm{GeV}$ for average water-air-crust nuclei) before the initial $X$ particle appreciably slows down or is deflected from its path. The effective penetration depth is then given by

$$
\ell_{p}=\lambda n_{\text {coll }}
$$

where $\lambda$ is the mean free path between succesive collisions given by

$$
\lambda=\frac{1}{n(A, Z) \sigma(X-(A, Z))} \approx \frac{10^{3} \mathrm{~cm}}{\sigma /(\text { Fermi })^{2}}
$$

where we used $n(A, Z)=N_{\text {Avagadro }} \rho /<m(A, Z)>$ and $\rho \approx 3 \mathrm{gm}^{-3}$, the average density of the crust of the earth. As a result the penetration length is given by $\ell_{p}=\lambda \frac{M_{X}}{20 \mathrm{GeV}} \approx \frac{10^{3} \mathrm{~cm}}{\sigma /(\text { Fermi })^{2}}\left(\frac{M_{X}}{20 \mathrm{GeV}}\right)$. Thus for $M_{X} \geq 2 \mathrm{TeV}$, the penetration depth is about a kilometer which is the typical depth for most underground Wimp detectors. Let us emphasize again that the key to the large number of the collisions, leading to large penetration depth is the small energy loss in any given X-nucleus collision due to the large mass of the $X$ particle. Even if the collision "drags" the nucleus to the same velocity $\beta_{X}$ of the $X$ particle, the kinetic energy of the nucleus will only be $\frac{1}{2} m(A, Z) \beta_{X}^{2}$ i.e. a factor $m(A, Z) / M_{X}$ smaller than the kinetic energy of the $X$ particle. Hence a large number of collisions $n_{\text {coll }}=M_{X} / m(A, Z)$ will be required to reduce its energy by a factor $e$ (i.e. $\left.\left(1-\frac{1}{n_{c 0 l l}}\right)^{n_{\text {coll }}} \equiv e^{-1}\right)$.

A somewhat more careful estimate of the $X$-nucleus cross-section $\sigma(X-(A, Z))$ can be obtained as follows. It has been argued before 14] that the slow $X G$ (where $G$ stands for the gluon) color neutral bound state, to which we will continue to refer to as the $X$ particle, sees the nucleus as a potential well of radius $R(A)$ and depth of order $V_{0} \simeq 10$ $\mathrm{MeV}$. The scattering is predominantly in S-wave. For the relative motion with reduced mass $m \simeq m(A, Z)$, energy $\approx \frac{m(A, Z) \beta^{2}}{2} \approx 10 \mathrm{keV}$ and momentum $p \simeq m(A, Z) \beta \approx 20 \mathrm{MeV}$ (assuming virial velocities for the particles) one gets a phase shift

$$
\delta_{0} \simeq-p R+\arctan \left(\frac{p}{\sqrt{p^{2}+2 m V_{0}}} \tan \sqrt{p^{2}+2 m V_{0}} R\right) \approx-p R
$$

where we have used the fact that $\sqrt{2 m V_{0}} R \gg 1$ so that the cross- section is essentially geometric i.e.

$$
\sigma \approx \pi R^{2} \approx \pi A^{2 / 3}(\text { Fermi })^{2}
$$

Using this one gets via Eq. 13,14 and 16 a penetration depth $\ell_{p} \approx 100$ meters in the rock for $M_{X} \approx 2$ TeV, confirming the earlier estimate.

If the above analysis applies, then the "wimp" detectors would be able to detect the full flux of the $X$ particles if located at appropriate depths where the enrgy attenuation is very small. Whereas most underground detectors (e.g. [8]) are located at greater depths, some are relatively shallow ( [7]) and will suited for this search. Note further that since the $X$-nuclear cross-sections are about $\approx 10^{12}$ times bigger than the generic heavy Dirac neutrino cross-section, if the local $X$ particle density is either given by the estimated halo density above or even the much smaller relic density, the detectors that ruled out the heavy Dirac neutrinos would a-fortiori also rule out the heavy $X$ particle.

We should note that the $X$ particles can be captured in nuclei once the center of mass collision kinetic energy $\frac{1}{2} M_{X} \beta^{2}$ is smaller than the expected few MeV X-nuclear binding energy i.e. $\beta_{X} \leq \sqrt{\frac{10^{-2} \mathrm{GeV}}{m(A, Z)}} \approx \frac{1}{50}$ which is satisfied for virial velocities. However once the nucleus is captured onto the $X$ particle, its crosssection (hence its attenuation 
length) will not necessarily increase (decrease) and in principle the nucleus could be dislodged in subsequent collisions. Hence any possible capture of the $X$ particle need not change the above estimate. However accompanying the capture of nucleus by the $X$, there could be some rather striking features as $\gamma$ or neutron emission which could serve as more sensitive signatures although it must be remembered that extra photon emission rate will down by a factor of $\alpha_{e m}$.

\section{B. Rare $X$-isotope formation}

We have estimated above that the relic cosmological abundance of $X$-paritcles relative to hydrogen is about $10^{-3}$ or so. Clearly this ratio need not be maintained locally on earth where we have a much enhanced baryon number density. To estimate the terrestrial $X$ particle effect, we first note that a flux $\Phi_{X}$ of such particles impinging the earth thru Hubble time yields a total of $\Phi_{X} \cdot t_{H} \simeq 2 \times 10^{17} \Phi_{X} X$-particles falling on each square centimeter on the earth. These $X$ particle would most likely accumulate in the ocean. For an ocean depth $\sim 10$ kilometers, and surface hydrogen density of $n_{H}=\frac{2}{18} N_{\text {Avagadro }} 10^{6}=10^{29} \mathrm{H} / \mathrm{cm}^{2}, r_{H} \equiv \frac{n_{X}}{n_{H}} \approx 10^{-12} \Phi_{X}$ and a similar number obtains for oxygen also. After slowing down to thermal velocities the $X$-particle will be captured by the nuclei in the ocean water- most likely the oxygen nuclei- forming anomalous heavy $\left(\approx M_{X}\right)$ isotopes. A careful search for precisely such heavy isotopes have been carried out in a recent experiment by Hemmick et al [6] and they find that the upper bound on the ratio $r_{\text {Oxygen }} \leq 4 \times 10^{-17}-3 \times 10^{-14}$ and $r_{H} \leq 2 \times 10^{-24}-3 \times 10^{-20}$ (for $M_{X}=0.1-10 \mathrm{TeV}$ ). Even if the collection period is shortened to 100 million years (a reasonable lifetime of the ocean), these upper bounds would seem to exclude $X$ particle fluxes $\Phi_{X} \geq 1 \mathrm{~cm}^{-2} \mathrm{sec}^{-1}$. If the $X$ 's constitute the dark galactic halo the expected flux $\Phi_{X}$ can be estimated to be

$$
\Phi_{X} \approx n_{X} v_{X}=\frac{0.3 \mathrm{GeV}}{M_{X} / \mathrm{GeV}} 3 \times 10^{7} \simeq 10^{3}-10^{4} \quad \text { for } \quad M_{X}=10-1 \mathrm{TeV}
$$

Thus $X$ as a halo dark matter is ruled out by the experiment in Ref. [6]. On the other hand, if $X$-particles do not dominate $\rho_{\text {cosmos }}$ and do not cluster in the Halos, so that Eq. 8 applies, then $\Phi_{X} \approx n_{X} v_{X} \approx 6 \times\left(10^{-3}-10^{-2}\right)$ which given the crudeness of our estimate is marginally consistent with the above lower bound.

\section{Annihilation in the Halo}

In this section, we consider the possibility that if the $X$ particle is its own antiparticle or the $X$ and $\bar{X}$ are present in the halo in equal abundance, their annihilation can give rise to energetic $\gamma$-ray fluxes. Present bounds on such fluxes can then lead to constraints on the $X$ particle density $n_{X}$. We derive this below.

We pointed out in section 2 that at small velocities $\beta \approx 10^{-3}$, where the $X$ energies are below $\Lambda_{Q C D}$, the $X-\bar{X}$ annihilation is enhanced and is given by $\sigma_{X \bar{X}} \approx \frac{1}{\beta_{X}}(\text { Fermi })^{2}$. The $X$ particles in the halo will then annihilate at a rate

$$
\frac{d n_{X} / d t}{n_{X}}=n_{X} v_{X} \sigma_{X \bar{X}} \approx n_{X}(F e r m i)^{2} c
$$

with $n_{X} \approx \frac{0.3 \mathrm{GeV}}{M_{X} / \mathrm{GeV}} \approx 10^{-4} \mathrm{~cm}^{-3}$ for $M_{X}=3 \mathrm{TeV}$. This implies a halo lifetime of $10^{20}$ sec., considerably exceeding $t_{H}$. As a result, only a fraction $\approx 10^{-3}$ of the initial $X$ 's will annihilate in Hubble time. Thus any galaxy will lose $\approx 10^{-3}$ of its rest mass via such annihilation. This exceeds the total energy output of stars.

The problem however is much more severe. The stellar radiation is largely carried away by the optical photons. whereas roughly 40 per cent of the energy released in $X-\bar{X}$ annihilation will convert to $100 \mathrm{GeV}$ to TeV photons. There are extremely strong bounds on these fluxes on earth [15]:

$$
\Phi_{\gamma} \leq 10^{-5} \mathrm{~cm}^{-2} \sec ^{-1} \mathrm{sr}^{-1}
$$

Since $\Phi_{\gamma} \simeq n_{X}^{2} \sigma_{X \bar{X}} v_{X} L$, Eq. 9 implies $n_{X} \leq 10^{-6} \mathrm{~cm}^{-3}$ where we have used $L \simeq 9$ kilopersecs as a typical halo radius.

In addition, these decays will also inject a large number of relativistic $e^{+} e^{-}$which if trapped in the galaxy will have an energy density of $\sim 0.3 f \mathrm{GeV} \mathrm{cm}^{-3}$ where $f \simeq 10^{-3}$ is the decaying fraction. Using equipartition of energy, this should not exceed the energy density in the galactic field which can be estimated to be $u_{B} \simeq \frac{\left(3 \times 10^{-6} \mathrm{Gauss}\right)^{2}}{8 \pi}=0.2 \mathrm{eV} \mathrm{cm}^{-2}$. 
However, a naive estimate of $u_{e^{+} e^{-}}$gives $\approx 3 \times 10^{-4} \mathrm{GeV} \simeq 3 \times 10^{5} \mathrm{eV}$ which is $10^{6}$ times larger. Thus both these arguments would imply that the halo density cannot be dominated by the massive $X$ particles. For unclustered $X$-particles, the analog of Eq. 19 with $L$ the effective halo size replaced by the Hubble radius, we find $n_{X} \leq 10^{-9}$ $\mathrm{cm}^{-3}$ (ignoring small redshift effects). Note that this is comparable to the cosmological prediction for $n_{X}$ in Eq. 8 .

\section{MASSIVE COLORED PARTICLES AS THE SOURCE OF UHE COSMIC RAYS}

During the past decades cosmic ray events with energies greater than $10^{20} \mathrm{eV}$ have been observed 11 in several independent experiments. This may call for new physics since cosmic ray proton with $E \geq 5 \times 10^{19} \mathrm{eV}$, scatter off the cosmic microwave background producing nucleon resonances leading to the Griesen-Zatsepin-Kuzmin cutoff. Moreover, the mean free path for this interaction is about $6 \mathrm{Mpc}$ (or redshift $z=0.0125$ ). Since such energetic protons are unlikely to be bent by the galactic magnetic fields their direction will point directly to the source which must be less than $6 \mathrm{Mpc}$ in distance if protons are to be the source of these UHE showers. No such source has been found [16]. On the other hand in the same general direction, there are some viable sources at greater distances (e.g. the quasar 3C 147 at a distance of 240 Mpc or the Seyfert galaxy MCG 8-11-11 at a distance of about $10 \mathrm{Mpc}$ ). For the primaries to be coming from these distant sources, they must avoid the analog of the GZK cutoff and must be strongly interacting to produce showers [10]. Therefore electrically neutral, colored particles are natural candidates for the primary particles. Furthermore, in order to successfully reproduce the observed extensive showers, these particles should have inelasticities comparable to those of the usual primary cosmic rays, namely protons or nuclei. In primary proton collisions, about half of the initial energy is utilized to make the energetic secondaries. The latter inelasticity is high. The arguments of the previous section implies that if the UHE primaries were heavy ( $\geq$ few hundred GeV) neutral colored particles they would have been seen in the underground detectors. Of course, if they are light (as in the model of Chung et al. [10]), our arguments do not apply. We now turn to the suggestion that heavy monopoles of grandunified theories may explain the UHE cosmic ray showers [12].

\section{MONOPOLES AS SOURCE OF ULTRA HIGH ENERGY COSMIC RAYS}

It was suggested in Ref. 12 that monopoles of grand unified theories may be responsible for the ultra high energy (UHE) cosmic rays with energies above $10^{20} \mathrm{eV}$ [12]. The attractiveness of the suggestion arises from the fact that this offers a natural mechanism for acceleration of the particles to such ultra high energies by the galactic magnetic

fields. For natural values of the monopole charges expected in GUT theories i.e. $E_{M} \simeq \sqrt{N} q_{M} B_{G} L_{c o h} \simeq 10^{20} \mathrm{eV}$ where $q_{M}=q_{e} / 2 \alpha$ is the monopole magnetic charge, $N$ is the number of coherent domains traversed and $L_{c o h}$ is the typical length of a coherence domain expected to be of order 100 persecs. To mimic the observed cosmic ray events, the monopole mass $m_{M}$ must be less than $10^{10} \mathrm{GeV}$ since it must be relativistic. There is also a kinematically a maximal value for the inelasticity i.e. energy transferred from the monopole in the scattering process on a target. To obtain this the authors of Ref. 7 note that the maximum energy transferred in a collision of a monopole of mass $m_{M}$ and energy $E_{M}$ on a target nucleon of mass $m$ is given by:

$$
\zeta_{\max }=\frac{2 m E_{M}}{\left(2 m E_{M}+m_{M}^{2}\right)}
$$

obtained for backward center of mass elastic scattering. We see that for UHE case of $E_{M} \simeq 10^{12} \mathrm{GeV}, \zeta$ acquires its maximal value $(\zeta=1 / 2)$ for $m_{M} \sim 10^{6} \mathrm{GeV}$ and decreases for higher values quadratically. If this maximum value for the inelasticity were attained, it would be very favorable for the interpretation of the monopoles as the source of UHE's. To see if this maximal inelasticity value can in fact obtain, one has to consider the dynamics of the monopole collision. We find that such high values for the inelasticity are unlikely to be realized in practice if either one of the two collision mechanisms that we consider dominates. Below we describe these two monopole-nucleus collision mechanisms and estimate the reaction rates and the inelasticities in both cases.

\section{A. Hard electromagnetic scattering of monopoles}

The most obvious reaction mechanism for initiating the UHE events by the monopoles utilizes their magnetic interaction with charged particles. The dominant mechanism briefly alluded to in Ref. [12] involve nuclear dusruption due to the Lorentz boost generated large transverse $E$-field seen by the stationary nucleus: 


$$
E_{T}=\frac{\beta \gamma q_{M}}{2 \alpha r^{2}} \approx \frac{\gamma q_{M}}{2 \alpha b^{2}}
$$

with $b$ being the impact parameter and $\gamma=\frac{E_{M}}{m_{M}}=10^{12} / m_{M}$ in $\mathrm{GeV}$. This field can disrupt the nucleus by accelerating the protons (relative to the neutrons). Let us estimate how big this effect is.

The external perturbation seen by the stationary nucleus is:

$$
H^{\prime}(t)=e \mathbf{E}_{T} \cdot \Sigma \mathbf{r}_{\mathbf{i}} f(t)
$$

where $\mathbf{r}_{\mathbf{i}}$ is the $i$ th proton's location relative to the center of the nucleus and $f(t)$ is a pulse shape normalized as

$$
\int f(t) d t \simeq \Delta t \simeq \frac{b}{\gamma c}
$$

where $b$ is the impact parameter and the $1 / \gamma$ factor is due to squeezing towards the transverse direction of the $B$ and $E$ field lines of the moving monopole. This leads to a $\gamma$ independent product $E \Delta t$. This product in turn controls both the boost invariant transverse momentum imparted $\sim F \Delta t$ and the excitation probability. The probability of exciting the nucleus is given in first order time dependent perturbation theory by the following sum over all non-ground states:

$$
\begin{aligned}
P=\Sigma P_{0 \rightarrow n} \leq e q_{M} Z^{2} E^{2} \Sigma_{n=1} \int\left|f(t) e^{i \Delta E_{n} t} d t\right|^{2} \mid & <0\left|\mathbf{r}_{\mathbf{i}}\right| n>\left.\right|^{2} \\
\leq(Z E)^{2}\left|\int f(t) d t\right|^{2}<0\left|r^{2}\right| 0> & \approx Z^{2}(E \Delta t)^{2} \bar{R}^{2} \\
& \approx Z^{2} \bar{R}_{\text {nucl. }}^{2} / b^{2}
\end{aligned}
$$

where Schwarz inequalities, completeness and $e q_{M}=1$ have been used. Thus only if $b \leq Z \sqrt{\bar{R}_{\text {nucl. }}^{2}} \approx$ $Z \times 1.2 A^{2 / 3} \mathrm{Fermi} \approx 60 \mathrm{Fermi}$, will we have $P \simeq 1$. The excitation cross-section is therefore smaller than $\sigma_{\text {dis }} \approx \pi b^{2} \approx 10^{-22} \mathrm{~cm}^{2}$. Furthermore, in each disruption, the total energy loss in the excited system rest frame does not exceed the total nuclear binding energy i.e. $\Delta E^{*} \leq 100 \mathrm{MeV}$. This gets boosted at most to $\Delta E=\gamma \Delta E^{*}$ in the laboratory frame. Even in this extreme case the fraction of the initial monopole energy lost in $\mathrm{GeV}$ in a collision is

$$
\eta \leq\left(\gamma m_{M}\right)^{-1}\left(\gamma \Delta E^{*}\right)=\Delta E^{*} / m_{M}=0.1 \mathrm{GeV} / m_{M}
$$

Along the line of sight in the atmosphere of average length $\ell \approx 20 \mathrm{~km}$, there are at most $N_{c}=\ell n_{N i t r o g e n} \sigma_{\text {dis }} \leq 10^{4}$ such collisions and the total energy loss is therefore $N_{c} \eta \simeq 10^{3} / m_{M}$, which is very small indeed for values of the monopole mass of interest.

Let us next consider the "hard collision" of a point like monopole with a point like charge. For a classical collision at impact parameter $b$, the boost invariant transverse momentum transfer is given by $\Delta p_{T}=e E_{T} \Delta t \approx \frac{e q_{M}}{b^{2}} b=e q_{M} / b$, where as discussed above $E_{T}=B_{T} \gamma \simeq e q_{M} \gamma / b^{2}$ and $\Delta t \approx b / \gamma$. This is precisely the same as the momentum transfer in a hypothetical Rutherford scattering with charges $2 Z_{1}=e$ and $Z_{2}=q_{M}$. Carrying this analogy to the field theoretic domain, we write down the Rutherford like cross-section for magnetic monopole and charge scattering as:

$$
\left.\frac{d \sigma}{d t}\right|_{q_{M}, e}(t) \simeq \frac{\left(q_{M} e\right)^{2}}{t^{2}}
$$

Although formally this leads to a divergent total cross-section, the invariant transfer in our case is simply $2 m T$ where $m$ is the mass of the electrically charged particle and $T$ is the laboratory energy transferred to it. The effective cross-section for monopole scattering obtained by weighing $\frac{d \sigma}{d t}$ with the inelasticity $T / E_{M}=t / 2 m E_{M}$ is given by

\footnotetext{
${ }^{2}$ It is amusing to recall that in nonrelativistic classical mechanics there is an exact description of the motion of a point charge in the field of a monopole. It is a convergent and a reflected spiral restricted to the Poincare cone with vertex at the origin and opening angle $\pi-\theta_{0}$ with $\theta_{0} \approx e g / L=e g / p b$, the ratio of the electromagnetic to the orbital angular momentum, such that when the cone is opened into a plane, the spiral becomes a zigzaging straight line [17]. The actual final scattering angle is $\theta \approx \theta_{0}$ and is just the same as for a Coulomb problem with $Q=g_{M}$ at the origin. Independently of all the above, we can compare the scattering amplitudes for charge-charge $(e-e)$, monopole-monopole $\left(q_{M}-q_{M}\right)$ and charge -monopole scattering $\left(e-q_{M}\right)$ via the inequality $A_{e-e} A_{q_{M} q_{M}} \geq A_{e-q_{M}}^{2}$.
} 


$$
\sigma_{e f f}=\frac{q_{M}^{2} e^{2}}{E_{M}} \int \frac{t}{2 m} \frac{1}{t^{2}} d t \approx \frac{q_{M}^{2} e^{2}}{2 m E_{M}} \ln \left(t_{\max } / t_{\min }\right)
$$

For $E_{M}$ of order $10^{12} \mathrm{GeV}$, this gives a cross-section for monopole scattering of magnitude $10^{-40} \mathrm{~cm}^{2}$, which is utterly negligible.

\section{B. Nuclear interaction of monopoles}

The magnetic monopole could have a hadronic cloud consisting of gluons and $q \bar{q}$ pairs. This can happen for example if the gauge group whose breaking leads to the monopoles includes $S U(3)_{c}$ as a subgroup. The hadronic cloud will then have a typical dimension $\sim \Lambda_{Q C D}^{-1}$ of order one Fermi or so. The hadronic cloud is inherent to the monopole and is expected to regenerate after every collision. Thus the monopole will have a hadronic cross-section for interaction with nuclei similar to that of a proton. This feature would seem to favor this scenario for understang the UHE events. In particular, one event from the Yakutsk experiment appears to be neutron rich suggesting a hadronic origin for the UHE's.

Unfortunately there is a crucial difference between the inelasticities of a monopole initiated and proton initiated UHE reaction. The hadronic outer layer of the monopole carries only a miniscule fraction $\epsilon \equiv \mathrm{GeV} / \mathrm{m}_{M}$ of the monopole mass. This can be seen for the 't Hooft-Polyakov monopole, where the bulk of the monopole mass $M_{M} \approx M_{G} / g_{G}$ (where $M_{G}$ and $g_{G}$ are the symmetry breaking scales and the gauge couplings associated with the GUT group whose breaking causes the appearance of the monopoles) resides in the innermost regions of the monopole (of dimension $\left.M_{G}^{-1}\right)$ and an order $\mathrm{TeV}$ contribution from the weak layer of the scale dimension $m_{W}^{-1}$. The extended hadronic layer contributes only a fraction $\Lambda_{Q C D} / g_{s} \approx G e V$ to the monopole mass. In the language of the parton model, the gluons and the $q \bar{q}$ pairs are the "wee" partons in the very small x-region of the parton distribution in the monopole. Since only thses hadronic constituents can participate in the hadronic monopole nucleus collision, we expect the inelasticity to be of order $\zeta \leq x \leq \mathrm{GeV} / m_{M}$. This result is clearly in contrast with the characteristic inelasticities of order $1 / 2$ in proton nucleus collision.

Possible binding of the monopole to the nuclei is also not likely to be important.The reason for this is that the binding potential is given by

$$
V=-\frac{\mu \cdot \mathbf{r}}{r^{2}} g_{M}
$$

where $\mu$ is the magnetic moment of the nucleus and $g_{M}$ is the magnetic charge of the monopole. Due to relatively small magnetic moment of the Oxygen and Nitrogen nuclei, it is not clear whether these nuclei can sustain the requisite p-wave monopole-nucleus bound state [18]. Even if such a bound state formed, it would strip off in the first nuclear collision and the rate of monopole nuclear capture to reform the composite is likely to be slower than that of the nuclear reactions destroying it. Hence the mechanism of enhancing nuclear monopole interaction by binding them to nuclei and thus enhancing $x_{H}$ from $G e V / m_{M}$ to $x_{H} \approx A(G e V) / m_{M}$ would be rather inefective. It turns out also that endowing the monopoles with large cross-sections to catalyze baryonic decays a-la Callan and Rubakov does not help either[

Thus in summary, we have shown that massive, stable and neutral colored particles with masses more than a few hundred $\mathrm{GeV}$ are ruled out by exisiting WIMP searches and masses in the range $100 \mathrm{GeV}$ to few TeV by the present results on searches for anomalous heavy isotopes. They are also not suitable for explanation of the UHE cosmic rays. Furthermore, as far as the monopoles are concerned, while we have not conclusively established that their interactions cannot lead to the UHE's, we have raised some plausible doubts about the effectiveness of the mechanism due to very small inelasticities in monopole nuclear interaction which can be inferred from simple intuitive arguments. To clearly

\footnotetext{
${ }^{3}$ While this general issue has been discussed in the past (see e.g. 19), the following argument may still be worth presenting. Let us consider a blue or red giant star of radius $R \approx 3 \times\left(10^{12}-10^{13}\right) \mathrm{cm}$. and column density of $\approx \frac{10 M_{\odot}}{\pi R^{2}} \approx 10^{8}-10^{6} \mathrm{gr} / \mathrm{cm}^{2}$. Clearly if the monopoles lose even $10^{-3}$ of their energy in our atmosphere, they would stop upon hitting these objects. During its lifetime of 10 million years, the number of monopoles accumulating there is $N_{M} \approx 4 \pi^{2} \Phi_{M} R^{2} T \approx \Phi_{M} 10^{42 \pm 1}$. After the gravitational collapse to a neutron star, most of these monopoles will reside in the nuclear core catalyzing one nuclar decay in each hadronic time scale of $10^{-23}$ sec.. Altogether in $3 \times 10^{16}$ sec., each monopole catalyzes $\approx 3 \times 10^{39}$ decays destroying a fraction $10^{-18}$ of the core. To allow the neutron stars to live for a billion years, we must require that $N_{M} \leq 10^{18}$ implying that $\Phi_{M} \leq 10^{-24 \pm 1} \mathrm{~cm}^{-2} \mathrm{sec}^{-1} \mathrm{sr}^{-1}$. This would totally exclude the above scenario.
} 
confirm or rule out the mechanism, detailed simulation of the effect of relativistic monopole air nuclei collision in the various experimental setups is required. Our net conclusion is that the monopole scenario for the UHE's is rather unlikely.

Acknowledgements The work of R. N. M. is supported by the National Science Foundation grant no.

PHY-9421386 and the work of both R. N. M. and S. N. is supported in part also by a grant from the Bi-national US-Israel National Science Foundation.

[1] A. de Rujula and S. L. Glashow and U. Sarid, Nucl. Phys. B333, 173 (1990).

S. Dimopoulos, D. Eichler, R. Esmailzadeh and G. Starkman, Phys. Rev. D 41, 2388 (1990);

A. Gould, B. Draine, R. Romani and S. Nussinov, Phys. Lett. B 238, 337 (1990).

[2] G. Farrar, Phys. Lett. B265, 395 (1991); Phys. Rev. Lett. 53, 1029 (184); 76, 4111 (1996).

[3] Z. Chacko, B. Dutta, R. N. Mohapatra and S. Nandi, hep-ph/9704307.

[4] S. Barr, D. Chang and G. Senjanović, Phys. Rev. Lett. ; Z. Berezhiani and R. N. Mohapatra, Phys. Rev. D 52, 6607 (1995); R. Foot and R. Volkas, Phys. Rev. D 52, 6595 (1995).

[5] G. Starkman, A. Gould, R. Esmailzadeh and S. Dimopoulos, Phys. Rev. D 41, 3594 (1990).

[6] T. Hemmick et al. Phys. Rev. D 41, 2074 (1990).

[7] D. Caldwell et al. Phys. Rev. Lett. 61, 510 (1988).

[8] F. Avignone et al. Phys. Rev. C 34, 666 (1986).

[9] V. Kuzmin, talk at NANP97, Dubna, July 7-11 (1997);

[10] D. Chung, G. Farrar and E. Kolb, FERMILAB-PUB-97/187-A; We thank A. Riotto for pointing out that other massive colored stable particles may be of interest in this connection.

[11] For a recent review, see M. Nagano, Nucl. Phys. B (Proc. Suppl.)52 B, 71 (1997).

[12] T. Kephart and T. Weiler, Astropart. Phys. 4, 271 (1996).

[13] See for instance, E. W. Kolb and M. Turner, Early Universe, Addison-Wesley (1989).

[14] S. Nussinov, hep-ph/9610236.

[15] M. Turner, quoted in E. W. Kolb, FERMILAB-Conf.-86/146-A.

[16] J. Elbert and P. Sommers, astro-ph/9410069.

[17] O. Kenneth, private communication.

[18] A. S. Goldhaber, S. Nussinov and L. Stodolsky, Nucl. Phys. B290, 955 (1988).

[19] S. Dimopoulos, J. Preskill and F. Wilczek, Phys. Lett. B 119, 320 (1982). 